\title{
On-line dispersion estimation and correction scheme for the Compact Linear Collider
}

\author{
J. Pfingstner ${ }^{*}$ and E. Adli \\ Department of Physics, University of Oslo, N-0371 Oslo, Norway \\ D. Schulte
}

CERN, Geneva 23 CH-1211, Switzerland

(Received 29 March 2016; published 24 January 2017)

\begin{abstract}
The Compact Linear Collider (CLIC) has stringent component alignment tolerances in order to preserve the ultralow emittance of the utilized particle beams. Beam-based alignment techniques have been designed to relax these tolerances to realizable values. In this paper, a scheme is presented that is capable of mitigating besides the effects of static misalignments also dynamic misalignments caused by ground motion. It is based on the well-known dispersion-free steering (DFS) algorithm, with the peculiarity that it can perform its correction during the usual operation (on-line). This is enabled by performing the necessary dispersion measurements by introducing only negligibly small beam energy changes (per mille level). It has been found that this on-line correction becomes sensitive to the imperfections of transverse wakefields and structure tilts. These sensitivities have been studied via analytical models and in simulations and appropriate countermeasures to improve the robustness of the method have been proposed. The correction performance and robustness properties of the improved algorithm have been studied in detail with respect to all relevant static and dynamic imperfections in a realistic scenario. The presented scheme is not only a potentially important operational tool for CLIC, but the findings with respect to robustness properties for different imperfections are of general interest for the application of the dispersion-free steering algorithm.
\end{abstract}

DOI: $10.1103 /$ PhysRevAccelBeams.20.011006

\section{INTRODUCTION}

The Compact Linear Collider (CLIC) [1] has very stringent alignment tolerances for its elements. These tight tolerances are needed to transport the particle beams with ultralow emittance without increasing their quality significantly [2-4]. The nominal horizontal and vertical emittances at the entrance of the main linac are only $\epsilon_{x, 0}=600 \mathrm{~nm}$ and $\epsilon_{y, 0}=10 \mathrm{~nm}$, respectively. The alignment tolerances can be relaxed by steering the beam by corrector magnets through the center of the beam position monitors (BPMs). But this measure is not sufficient, and additional beambased alignment techniques are indispensable.

There have been significant achievements in the development of such beam-based alignment techniques [5], especially for the Stanford Linear Collider (SLC) [6] at SLAC. The currently most prominent scheme is the dispersionfree steering (DFS) algorithm [7]. It has been studied analytically as well as in simulations for several different machines with great success, e.g., for SLC [8], the International Linear Collider (ILC) $[9,10]$, and CLIC

\footnotetext{
*juergen.pfingstner@fys.uio.no

Published by the American Physical Society under the terms of the Creative Commons Attribution 3.0 License. Further distribution of this work must maintain attribution to the author(s) and the published article's title, journal citation, and DOI.
}

[11]. The technique has also been applied to lattices with nonlinear magnets [12], and generalizations have been proposed, which take into account wakefield effects [13]. The algorithm has been tested experimentally at SLC [14], LEP [15], and FACET [16,17].

The DFS algorithm will be applied for the initial setup of the CLIC main linac [18] and after realignment campaigns, which are performed in shutdown periods. Simulations studies show, however, that dynamic component misalignments, e.g. due to ground motion, cause also an increase of the beam emittance during the operation on a short time scale. As a result, the DFS correction will have to be reapplied regularly, which will cause additional machine downtime.

The following simulation results quantify this statement. The simulations have been performed with the simulation setup described in Sec. II E for the main linac of CLIC. Ground motion according to the ATL-law [19] has been applied to the beamline, where a site dependent constant $A$ of $10^{-5} \mu \mathrm{m}^{2} \mathrm{~m}^{-1} \mathrm{~s}^{-1}$ has been assumed. After the misalignment, a beam steering has been performed, which approximates the action of a well designed and continuously running orbit feedback. The final emittance growth $\Delta \epsilon_{y}$ has been evaluated for 20 random seeds as a function of time for the more sensitive vertical direction. The results show that the emittance with a time constant of $7.5 \%$ per hour. This corresponds to doubling of the initial emittance $\epsilon_{y}$ of 
$10 \mathrm{~nm}$ in only 13 hours, which shows that the DFS correction has to be applied at least on an hourly basis. This causes an operational problem, since the conventional DFS algorithm cannot be applied during the normal operation. The physics run has to be stopped to perform the correction, which increases the downtime significantly. The limitation originates from a dispersion measurement necessary to compute the DFS corrections, where relatively large beam energy changes have to be introduced (see Sec. II A for more details), which is not possible during the usual operation.

In this paper, a specific way of measuring the dispersion for the DFS algorithm is proposed to overcome this limitation. By introducing only very small energy changes (per mille level), the dispersion measurement can be performed in parallel with the usual accelerator operation (on-line) without causing disturbances. The maximum allowable energy change is determined by the energy acceptance of the beam delivery system (BDS). For energy variations $<0.1 \%$ the corresponding luminosity loss is $<1 \%$ (see [1], p. 196). The created energy variations are also of no problem for the physics analysis, since the variations are measured in the BDS with high precision and can be corrected for if necessary. The drawback of the smaller induced energy variations is a higher sensitivity with respect to machine imperfections. Hence, the robustness of the dispersion measurement and correction has been studied for all relevant imperfections in this paper. Three especially critical imperfections have been identified and are analyzed separately. Beside the obvious problem of BPM noise (Sec. II B), also transverse wakefields (Sec. III) and structure tilts (Sec. IV) have turned out to cause a significant performance degradation. However, the robustness properties of these imperfections can be significantly improved by modification of the basic dispersion measurement procedure. Considering these modifications, different realistic implementation scenarios are proposed and tested in Sec. V. For the most promising scheme, more detailed performance and robustness studies are presented in Sec. VI.

\section{ON-LINE DISPERSION ESTIMATION AND CORRECTION SCHEME}

\section{A. Dispersion-free steering algorithm}

The principle of the emittance growth due to component misalignments is the following. If the beam passes through quadrupole magnets with an offset, it is not only exposed to the expected quadrupole field, but also to an unwanted dipole field. The resulting dipole kick induces beam oscillations along the beamline. The orbit correction reduces these beam oscillations. But since also the BPMs are misaligned, the beam is still not steered through the centers of the quadrupole magnets which results in residual dispersion. This dispersion in combination with

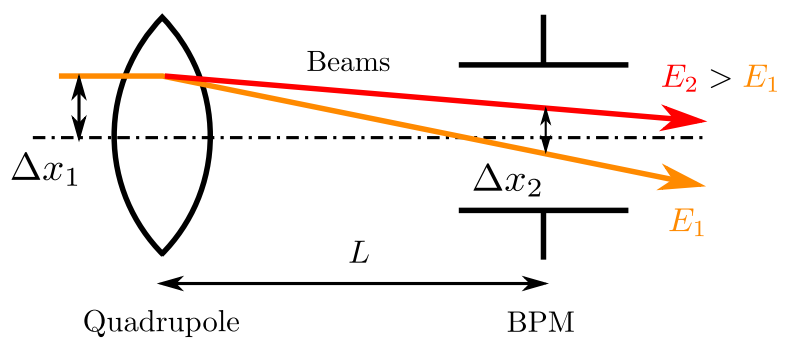

FIG. 1. Illustration of the basic principle of dispersionfree steering, where two beams with the same offset $\Delta x_{1}$ in a quadrupole magnet are deflected differently, if they have different beam energies $E_{1}$ and $E_{2}$.

the large beam energy spread particles leads ultimately to an increase of the projected emittance via a phase mixing of particles with different energy which is called filamentation.

The DFS algorithm cures this problem by moving the quadrupole magnets (or alternatively changing the strengths of corrector dipole magnets) in a way such that the resulting beam orbit has lower dispersion values. The basic principle to find such a dispersion-free orbit is illustrated in Fig. 1. If a beam passes through a quadrupole magnet exactly in its center, a change of beam energy will not result in a beam position change in a downstream BPM. If, however, the beam is offset by a distance $\Delta x_{1}$, the beam is deflected by the dipole kick from the quadrupole magnet, which is proportional to $\Delta x_{1}$. A second test beam with a higher energy $E_{2}$ is less deflected by the quadrupole, due to the higher beam rigidity. The deflection difference $\Delta x_{2}$ in the downstream BPM is therefore a direct measure for the dispersion in the beamline. This dispersion measurement can be used together with the known beam energy change, lattice geometry, and quadrupole magnet strength to calculate the beam offset $\Delta x_{1}$. The quadrupole magnet can then be moved by $\Delta x_{1}$ to minimize the dispersion. Clearly, this procedure will be disturbed by BPM noise.

If this concept is generalized from one quadrupole magnet and one BPM to a whole beamline, the necessary quadrupole magnet movements $\Delta \boldsymbol{\theta}$ can be found by solving the following system of linear equations in a least squares sense [12]

$$
-\left[\begin{array}{c}
\boldsymbol{x}-\boldsymbol{x}_{\mathbf{0}} \\
\omega\left(\boldsymbol{\eta}-\boldsymbol{\eta}_{\mathbf{0}}\right) \\
\mathbf{0}
\end{array}\right]=\left[\begin{array}{c}
\boldsymbol{R} \\
\omega \boldsymbol{D} \\
\beta \boldsymbol{I}
\end{array}\right] \Delta \boldsymbol{\theta},
$$

where $\boldsymbol{x}$ and $\boldsymbol{\eta}$ are vectors containing the measured beam orbit and dispersion, $\boldsymbol{x}_{0}$ and $\boldsymbol{\eta}_{0}$ are the reference orbit and reference dispersion. The matrices $\boldsymbol{R}$ and $\boldsymbol{D}$ are the orbit and dispersion response matrices. Their elements $r_{i j}$ and $d_{i j}$ characterize the first-order response of the beam orbit $x_{i}$ and dispersions $\eta_{i}$ in the $i$ th BPM, due to a change of the $j$ th corrector and are defined as 


$$
\begin{gathered}
r_{i j}=\left.\frac{\partial x_{i}}{\partial \theta_{j}}\right|_{\theta_{j}=0} \\
d_{i j}=\left.\frac{\partial \eta_{i}}{\partial \theta_{j}}\right|_{\theta_{j}=0} .
\end{gathered}
$$

By solving Eq. (1), not only the dispersion is minimized [middle part of Eq. (1)], but also the beam orbit is kept small [upper part of Eq. (1)]. The lower part of Eq. (1) is introduced to damp the actuations to prohibit solutions with too high corrector excursions. The relative importance of these three different objectives can be weighted with the parameters $\omega$ and $\beta$ that will be chosen later in this paper to optimize the correction result.

As explained above, the dispersion $\boldsymbol{\eta}$ is measured in a linac by using two beams with different energies $E_{0}(1+\delta)$ and $E_{0}$, where $E_{0}$ corresponds to the energy of the reference particle and $\delta \ll 1$. The corresponding BPM measurements $\boldsymbol{x}_{\delta}$ and $\boldsymbol{x}_{0}$ are used to compute $\boldsymbol{\eta}$ as

$$
\boldsymbol{\eta}=\frac{\boldsymbol{x}_{\delta}-\boldsymbol{x}_{0}}{\delta}
$$

The energy scaling $1+\delta$ can be created by a change of the amplitude or the phase of the acceleration voltage in the acceleration structures. Usually the input $\mathrm{rf}$ power is supplied by klystrons. In the case of CLIC, however, the two-beam concept has been adopted. Usually the relative beam energy change is created uniformly along the whole accelerator, which means that all accelerating gradients are scaled by $1+\delta$ as well as the initial beam energy. Such an energy change will be referred to as a global energy change, in contrast to a local energy change.

\section{B. On-line dispersion estimation}

For an on-line application of the DFS algorithm, the energy changes that can be introduced to measure the dispersion are limited to about $0.1 \%$ at the end of the main linac, due to the energy acceptance of the beam delivery system. This is significantly smaller than the usually introduced energy changes, which are in the order of 5\% to $10 \%$.

The problem that arises from these small energy variations is that the induced orbit changes for the dispersion measurement are small compared to the BPM resolution and the effects of other imperfections. To improve the situation, an averaging strategy is utilized. Many orbit measurements from beams with small energy changes $\boldsymbol{\delta}=\left[\delta_{1}, \delta_{2}, \ldots, \delta_{N}\right]^{T}$ are combined to one dispersion measurement. If the vector of measured beam orbits $\boldsymbol{x}_{i}$ for the $i$ th $\mathrm{BPM}$ is written as

$$
\boldsymbol{x}_{i}=\eta_{i} \boldsymbol{\delta}+x_{i}^{(0)}+\boldsymbol{n}_{i},
$$

where $x_{i}^{(0)}$ is the reference orbit $(\delta=0)$ and $\boldsymbol{n}_{i}$ is white Gaussian BPM noise with a standard deviation of $\sigma_{\mathrm{BPM}}$, then the optimal estimate for $\eta_{i}$ and $x_{i}^{(0)}$ is a least squares estimate [20] given by

$$
\begin{gathered}
{\left[\begin{array}{c}
\eta_{i} \\
x_{i}^{(0)}
\end{array}\right]=\left(\Phi^{T} \Phi\right)^{-1} \Phi^{T} \boldsymbol{x}_{i},} \\
\Phi=\left[\begin{array}{cc}
\delta_{1} & 1 \\
\delta_{2} & 1 \\
\vdots & \vdots \\
\delta_{N} & 1
\end{array}\right] .
\end{gathered}
$$

A convenient choice for $\delta$ is

$$
\boldsymbol{\delta}=\left[\begin{array}{lllll}
-1 & 0 & -1 & \ldots & 0
\end{array}\right] \delta,
$$

which is motivated by the fact that it is easier to lower the beam energy than to increase it. For this case, Eq. (6) simplifies significantly to

$$
\begin{gathered}
x_{i}^{(0)}=\frac{2}{N} \sum_{k=1}^{N / 2} x_{i, 2 k}, \\
\eta_{i}=\frac{2}{\delta N} \sum_{k=1}^{N / 2}\left(x_{i, 2 k}-x_{i, 2 k-1}\right),
\end{gathered}
$$

where $k$ is the time index of the measurement data.

\section{Full correction strategy and practical implementation}

The DFS correction is part of the overall beam-based alignment scheme for the CLIC main linac. The full scheme has been implemented in simulations to evaluate the performance of the dispersion correction. It is illustrated in Fig. 2.

The beam steering algorithm [21] moves the quadrupole magnets of a beamline transversally to steer a beam in to the centers of all BPMs. The rf alignment [22] is a strategy to reduce transverse wakefields, which are a main challenge

\begin{tabular}{|l|}
\hline Beam steering \\
\hline rf alignment \\
\hline Linac corrections: 1 to $N_{i}$ \\
\hline \begin{tabular}{|l|}
\hline Bin corrections: 1 to $N_{b}$ \\
\hline Dispersion measurement \\
\hline DFS corrections calculation \\
\hline Application of corrections \\
\hline rf alignment \\
\hline
\end{tabular}
\end{tabular}

FIG. 2. Structure of the overall beam-based alignment scheme for the CLIC main linac. 
for the emittance preservation in CLIC. Therefore, socalled wakefield monitors (WFM) are integrated in the accelerating structures to measure directly the transverse wakefields created by the beam. This information is used to mitigate the transverse wakefields by realigning the accelerating structures to the beam. As will be explained in Sec. III, the on-line dispersion estimation is sensitive to transverse wakefields, and hence a rf alignment is performed initially, but also after each full dispersion correction of the whole beamline.

The dispersion correction of the linac is not performed in one step. Instead the linac is separated into $N_{b}$ partially overlapping bins that are corrected one after the other. For the main linac, $N_{b}$ has been chosen to be 36 . The binning improves the robustness with respect to the necessary system knowledge (response matrices). The overlap of the bins ensures a continuous transition from one corrected section to the next. Simultaneously with applying the found dispersion corrections, the orbit downstream of the corrected bin has to be controlled to avoid large beam excursions. This is achieved by predicting the downstream beam oscillations and correcting them with a few correctors located right after the corrected bin. The correction of the full linac is iterated $N_{i}$ times, which improves the results with respect to the wakefield sensitivity. Simulation studies have shown that a number $N_{i}$ of three iteration is sufficient to reach convergence.

\section{Parameter determination}

The presented form of the on-line DFS algorithm has four open parameters: $\omega, \beta$, the energy change $\delta$, and the number of averaged orbits $N$. The weight $\omega$ determines the relative importance of the dispersion correction in relation to the orbit correction and the strength of the corrector actuations. There exists theoretical optimum [7] given by

$$
\omega_{\mathrm{opt}}=\sqrt{\frac{\sigma_{\mathrm{O}}^{2}+\sigma_{\mathrm{BPM}}^{2}}{2 \sigma_{\mathrm{BPM}}^{2}}},
$$

where $\sigma_{\mathrm{O}}$ is the standard deviation of the misalignments of the BPMs around a straight line, and $\sigma_{\mathrm{BPM}}$ is the BPM resolution. This optimum value is, however, only valid for certain assumptions and therefore $\omega_{\text {opt }}$ is determined in this paper via simulations studies in Sec. V.

The parameter $\beta$ is used to reduce the size of the corrector actuations (quadrupole movements) in case of too large excursions. However, for all simulations performed in this work the corrector actuation were not a limiting factor, and hence the parameter $\beta$ could be set to 0 , which corresponds to unconstrained actuations. With this choice, the actuations have been less than $1 \mu \mathrm{m}$ RMS for all tested imperfections apart from BPM offsets, for which the actuations in the order of $20 \mu \mathrm{m}$ have been observed. As soon as $\beta$ was set to values that reduced these actuations by a significant fraction, the DFS correction performance was significantly worsened.

As already discussed, the energy change $\delta$ at the end of the linac has to be limited to about 1 per mille for an on-line application. The exactly used value depends on the specific scheme with which the energy change has been introduced (see Sec. V). But the value was always around 1 per mille or lower. For benchmarking reasons, some simulations have been also performed with $\delta \mathrm{s}$ as large as $5 \%$.

The number $N$ of used orbits for one dispersion estimate determines the effective BPM resolution $\tilde{\sigma}_{\mathrm{BPM}}$, which is defined here as

$$
\tilde{\sigma}_{\mathrm{BPM}}=\sqrt{\frac{2}{N}} \sigma_{\mathrm{BPM}}
$$

Considering Eq. (4), the remaining noise level in the estimated dispersion $\boldsymbol{\eta}$ is given by

$$
\tilde{\sigma}_{\eta}=\frac{\sqrt{2}}{\delta} \tilde{\sigma}_{\mathrm{BPM}} .
$$

The simulation studies have shown that for CLIC an $N$ of 100 is a good compromise, between an effective BPM resolution of $7.07 \mathrm{~nm}$ and the overall correction time $T_{c}$ of 4 minutes and 16 seconds, which can be calculated as

$$
T_{c}=\left(\frac{N}{f_{\text {rep }}} N_{b}+T_{r f}\right) N_{i}+T_{r f}
$$

In this expression, $f_{\text {rep }}, N_{b}$, and $N_{i}$ are the beam train repetition rate, the number of correction bins, and the number of linac correction iterations, respectively. The necessary time to perform a rf alignment $T_{r f}$ is about 10 seconds.

\section{E. Simulation setup}

All simulations in this paper have been performed with the tracking code PLACET [23]. The code includes realistic models of all relevant components of the main linac as accelerating structures, magnets and instrumentation hardware. Also, collective effects as longitudinal and transversal wakefields are included. The beam is modeled via 375 macro-particles that are arranged in 25 longitudinal slices, to allow a detailed simulation of the correlated and uncorrelated energy spread along the linac. PLACET already includes several beam-based correction techniques, e.g., one-to-one steering and rf alignment.

To facilitate the implementation of complex simulations scenarios, PLACET has been utilized in combination with the simulation framework LinSim [24]. This framework uses PLACET as a tracking tool, but simplifies the work of the user by supplying functionalities as lattice and beam setup for different machines, application of imperfections and data handling. The implemented code has been written in the OCTAVE [25] language. LinSim also allows us to apply 
different ground motion models to the beamline and to run orbit feedback systems in parallel to the dispersion measurement and correction.

\section{WAKEFIELDS AND DFS PERFORMANCE}

\section{A. Wakefield sensitivity}

When applying the DFS algorithm outlined in Sec. II C with $\delta$ in the per mille level to the main linac of CLIC, it becomes apparent that the correction performance is very sensitive to transverse wakefields. The problem of this strong sensitivity originates from dispersion that is created by the transverse wakefields itself. The initial rf alignment reduces the dispersion from wakefields, but cannot eliminate them completely due to the limited resolution of the wakefield monitors. The properties of the according wakefield dispersion will be studied in Sec. IIIC, where especially the differences to the dispersion due misaligned quadrupole magnets is worked out. This has been done with the help of an analytic model for the dispersion in a beamline due to a single dipole kick that is derived in Sec. III B and Appendix A. In Sec. III D, the effect of the wakefield dispersion on the DFS correction is analyzed and finally, a method to mitigate the DFS performance degradation is presented in Sec. III E.

\section{B. Model for dispersion in linear accelerators}

Since dispersion in a linear accelerator does not coincide with the intuitive picture of dispersion in a storage ring, an analytic model for the former is derived in Appendix A. In this section, the obtained expressions are interpreted and compared with simulations.

In this model, the dispersion $\eta(s)$ along a beamline due to a single dipole kick $\Delta x_{0}^{\prime}$ at location $s_{k}$ has been considered. It has been found in Eqs. (A10)-(A11) that for this case $\eta(s)$ is given by

$$
\begin{aligned}
\eta\left(s>s_{k}\right) & =\sqrt{\beta_{0}\left(s_{k}\right) \beta_{0}(s)} T(s) \frac{\Delta x_{0}^{\prime}}{\delta} \quad \text { with } \\
T(s) & =\sin \left[\hat{\phi}_{\delta}(s)\right]-\sin \left[\hat{\phi}_{0}(s)\right],
\end{aligned}
$$

where $\beta_{0}(s)$ is the beta function for a particle with nominal energy $E_{0}$ and $\hat{\phi}_{0}(s)$ is the phase advance measured from the kick location. The term $\hat{\phi}_{\delta}(s)$ is the phase advance for a particle with an energy $E_{0}(1+\delta)$.

This expression for $\eta(s)$ can be separated into a linear and a nonlinear regime depending on the phase difference $\Delta \hat{\phi}_{\delta}(s)$ between $\hat{\phi}_{\delta}(s)$ and $\hat{\phi}_{0}(s)$. For $\Delta \hat{\phi}_{\delta}(s) \ll 1, \eta(s)$ can be simplified to Eq. (A17)

$$
\eta_{L}(s)=\sqrt{\beta_{0}\left(s_{k}\right) \beta_{0}(s)} \cos \left[\hat{\phi}_{0}(s)\right] \frac{\xi_{F} \hat{N}_{q}(s)}{2} \Delta x_{0}^{\prime},
$$

where $\xi_{F}$ is the chromaticity of one FODO cell and $\hat{N}_{q}(s)$ is the number of quadrupoles between the kick location $s_{k}$ and $s$. In this linear regime, the dispersion $\eta_{L}(s)$ has the form of the usual betatron motion in Eq. (A3), but with a phase shift of $\pi / 2$ and amplitude modulated with a linearly rising function given by

$$
A_{\eta}(s)=\frac{\xi_{F} \hat{N}_{q}(s)}{2} .
$$

This linear dispersion $\eta_{L}(s)$ is independent of $\delta$ and hence coincides with the intuitive picture of dispersion in storage rings.

Further downstream, the dispersion becomes nonlinear. In this regime, the envelope function is not given by the linearly rising function $A_{\eta}(s)$ anymore, but the dispersion corresponds to the beating of the two sine functions in $T(s)$. The maximum amplitude of this beating dispersion wave is the amplitude of the betatron motion in Eq. (A3), namely $\sqrt{\beta_{0}\left(s_{k}\right) \beta_{0}(s)} \Delta x_{0}^{\prime}$, multiplied by the constant factor

$$
B_{\eta}=\frac{2}{\delta} .
$$

The smaller $\delta$, the larger is the dispersion in the nonlinear regime. This is due to the fact that the dispersion stays for a longer distance in the linear regime if $\delta$ is small, since $\Delta \hat{\phi}_{\delta}(s)$ grows slower, and hence rises to higher values.

These analytic results are reflected by the simulations shown in Fig. 3. For the first 100 quadrupole magnets after the dipole kick (one BPM per quadrupole magnet), the dispersion stays in the linear regime and is independent of $\delta$. Further downstream, a strong dependence of the shape and the amplitude of the dispersion is observable, which corresponds to the nonlinear regime. The predicted beating of the dispersion can be seen, as well as the dependence of the maximal amplitude on $\delta$. An effect that is not covered

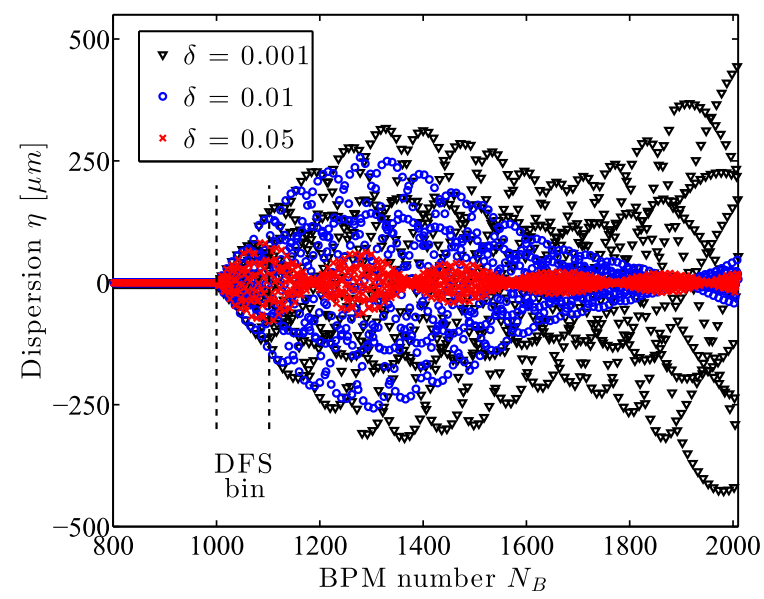

FIG. 3. Simulated dispersion due to a dipole kick created by the 1000th quadrupole magnet of the main linac of CLIC, which is vertically misaligned. Different energy changes $\delta$ result in different measured dispersion patterns, especially far downstream of the kick (nonlinear regime). 
by the analytic dispersion model, but is well modeled in simulations is the filamentation of the oscillations of the individual beams used for the dispersion measurement. It originates from the beam energy spread along of the beams used for the dispersion measurement. The beams enters the main linac with an uncorrelated energy spread of $2 \%$ and develops a mainly correlated energy spread of about $0.4 \%$ along the main linac, due to the off-crest acceleration and the longitudinal wake fields. As can be seen in Fig. 3, filamentation has a damping effect on the dispersion. This damping is stronger for larger $\delta$ than for smaller ones.

\section{Dispersion due to wakefields}

Both, misaligned quadrupole magnets and misaligned acceleration structures apply kicks to a passing particle beam, which creates dispersion in both cases. However, the dispersion patterns created by wakefields and quadrupole magnets are different. The reason for this behavior is that for a misaligned quadrupole magnet, the applied dipole field is constant for all particles of the beam, while for a transverse wakefield the field is stronger at the tail of the beam than at its head. The amplitude of the dispersion created by a wakefield is hence not uniform along the bunch, contrary to the dispersion from a misaligned quadrupole magnet. Still, the shape of the dispersion propagating along the linac would be the same for both cases if the particles in the head and the tail would oscillate in the same way. But in the CLIC main linac, the average particle energy is different for the head and the tail of the beam (off-crest acceleration, longitudinal wake fields), which causes the particles to have different dispersion patterns. As a result, the average dispersion patterns measured by the BPMs are different for kicks from a transverse wakefield and a misaligned quadrupole magnet.

To show the effect of the wakefield dispersion, the following simulation has been performed. Starting from a perfectly aligned beamline, a rf alignment has been carried out with the nominal wakefield monitor resolution of $3.5 \mu \mathrm{m}$. The limited resolution of the wakefield monitors causes the structures to be moved from the ideal position, which results in residual transverse wakefields that influence the dispersion correction. With this procedure, the quadrupole magnets stay aligned, which allows studying the influence of the wakefields on the dispersion independently. The dispersion has been calculated for different induced energy changes assuming perfect BPM resolution. The results are depicted in Fig. 4, where the dispersion from wakefields is clearly visible. Its amplitude grows along the linac as more and more wakefield kicks are accumulated by the beam. As analytically predicted in Eq. (16), the dispersion is stronger for smaller $\delta$, where a $\delta^{-1}$ scaling has been suggested. Additionally, the filamentation effects (not included in the analytical estimate) are stronger for larger $\delta$, which further decreases the accumulated dispersion.

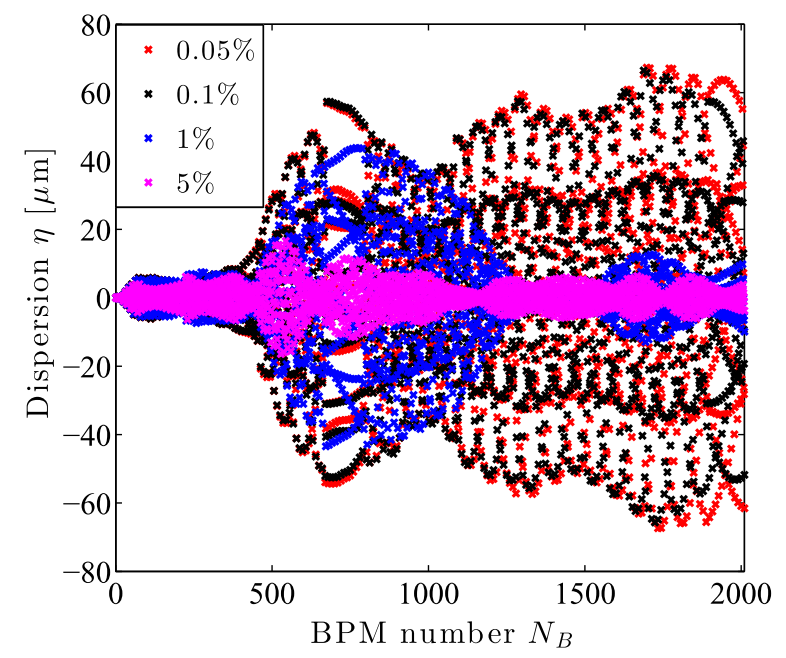

FIG. 4. Dispersion due to typical wakefields after rf alignment in the CLIC main linac for different global energy changes $\delta$ from $0.05 \%$ to $5 \%$ employed for the dispersion measurement.

\section{Effects of wakefields on the dispersion correction}

When the dispersion is measured, the DFS correction algorithm finds a solution in which the dispersion from wakefields is compensated via the dispersion from quadrupole magnets. However, the dispersion is only canceled on average, but there is still dispersion present along the beam that only adds up to zero. This is due to the fact that dispersion from a wakefield is not uniformly distributed along the bunch, while the dispersion from quadrupole magnets is. Hence the dispersion from a quadrupole magnet can never fully compensate the dispersion from a wakefield. Also, the cancellation on average is only valid for a corrected bin, but not further downstream, where the dispersion does not cancel on average anymore.

If only one bin would be considered the effect of wakefields could be neglected, since the dispersion values are small compared to the ones from misaligned quadrupole magnets. However, since the dispersion from wakefields is not fully cancelled, it accumulates from bin to bin and creates larger and larger dispersion signals in downstream bins. The DFS algorithm reacts on this signal and adds more and more dispersion from quadrupole magnets to compensate it on average. The dispersion that remains in the different beam slices causes an emittance growth and hence a performance degradation of the DFS algorithm. This is especially the case, in combination with the necessary final rf alignment, which changes the wakefields along the linac and destroys also the dispersion cancellation on average.

As has been pointed out above, dispersion from wakefields at the beginning of the linac will influence the correction in all downstream bins. The associated dispersion is not in the linear regime anymore, but in the nonlinear regime. Its amplitude is therefore larger for smaller $\delta$, as predicted in Sec. II B. On the other hand, dispersion from misaligned 
quadrupole magnets does not reach the nonlinear regime, since it is fully cancelled in the bin in which is created and does not travel further downstream. Hence the ratio of wakefield dispersion to quadrupole magnet dispersion gets larger for smaller $\delta$, which explains the energy dependence of the sensitivity to transverse wakefields.

\section{E. Countermeasures against wakefield sensitivity}

As will be shown in Sec. V, for the CLIC wakefield monitor resolution of $3.5 \mu \mathrm{m}$ and an induced energy change of 1 per mille, the DFS performance is degraded by the residual wakefields to an unacceptable level. The dispersion from wakefields can be lowered by increasing $\delta$. This is, however, not feasible for an on-line application of DFS. Another option would be more stringent specifications for the wakefield monitor resolution. But this would increase the cost of the structure fabrication strongly and can hence also be discarded.

Another solution can be anticipated, by observing that also wakefield dispersion stays in a linear regime, according to Eq. (15), if it is created shortly before the correction bin. In this regime, the dispersion is independent of $\delta$ and stays at relatively small values. This observation suggests that the wakefield sensitivity can be lowered if the energy change $\delta$ for the dispersion measurement is created only shortly before the DFS bin. This strategy has the additional advantage that fewer structures contribute to the creation of dispersion.

The advantage of such a local energy change compared to a global one is illustrated in Fig. 5. The same simulation setup as for Fig. 4 was used. While the wakefield dispersion with a global energy change is large in bin 25 , the wakefield dispersion created by a local energy change is much smaller

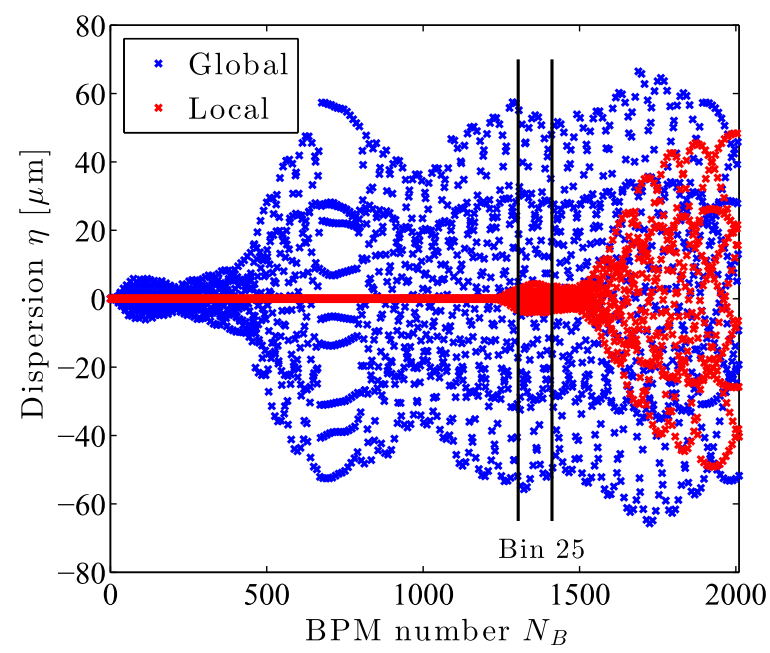

FIG. 5. Dispersion due to typical residual transverse wakefields after rf alignment in the CLIC main linac for global and local energy changes $\delta$ of 1 per mille. The local energy change is adjusted to correct bin 25 , which is marked in the plot with the two vertical black lines. in this area. The dispersion from the local scheme only grows to high values downstream of bin 25 (nonlinear regime). In this simulation, the local energy change has been introduced by changing the acceleration voltage in only 4\% (2986 $23 \mathrm{~cm}$ structures) of the main linac structures, namely the ones that belong to the decelerator located before bin 25 .

\section{STRUCTURE TILTS AND DFS PERFORMANCE}

In Sec. III, it was found that the wakefield sensitivity of DFS can be lowered if the energy change $\delta$ for the dispersion measurement is introduced shortly before the correction bin (local energy change). Simulation studies have shown, however, that for such a local scheme, the DFS algorithm becomes sensitive to tilts of the acceleration structures. This effect is so pronounced that the DFS performance becomes unacceptable (see Sec. V). In the following, this tilt sensitivity is explained with the help of an analytical model. The presentation of appropriate countermeasures will be postponed to Sec. V.

If an acceleration structure is tilted by an angle $\varphi_{s}$, a passing beam receives a transverse kick $\Delta x_{\varphi}^{\prime}$. This kick can be separated into the kicks $\Delta x_{\text {f,in }}^{\prime}$ and $\Delta x_{\text {f,out }}^{\prime}$ from the entry and the exit fringe field, and the kick $\Delta x_{E}^{\prime}$ due to the transverse component of the electric field as

$$
\begin{aligned}
\Delta x_{\varphi}^{\prime} & =\Delta x_{\mathrm{f}, \text { in }}^{\prime}+\Delta x_{E}^{\prime}+\Delta x_{\mathrm{f}, \text { out }}^{\prime} \\
& =\frac{G_{s} L_{s}}{E_{0}} \sin \varphi_{s}\left(-\frac{1}{4}+1-\frac{1}{4}\right) \approx \frac{G_{s} L_{s}}{2 E_{0}} \varphi_{s},
\end{aligned}
$$

where $L_{s}$ and $G_{s}$ are the length and the acceleration gradient of the structure, and $E_{0}$ is the beam energy given in $\mathrm{eV}$. In the last step, it has been assumed that $\varphi_{s} \ll 1$, which is the case for CLIC, where the specification for the tilt standard deviation $\sigma_{\varphi}$ is $140 \mu \mathrm{rad}$.

If uncorrected, the kicks from the structure tilts create large beam oscillations that increase the beam emittance strongly. These orbit excursions can be corrected by a simple beam steering, which already reduced the emittance growth $\Delta \epsilon_{y}$ to about $3 \%$ in the more sensitive vertical direction. Also, the remaining dispersion is insignificant and a consecutive dispersion correction does not result in a further reduction of $\Delta \epsilon_{y}$.

However, while structure tilt effects have only a small effect on the machine performance after beam steering, they can still degrade the consecutive DFS correction to an unacceptable level. This degradation is caused by a measurement artefact, which is not related to physical dispersion. In Appendix B, it is shown in Eq. (B3) that the measured dispersion $\eta$ due to a tilted structure is given by

$$
\eta_{\varphi}=\eta_{\varphi 0}+\eta_{\varphi A},
$$

where $\eta_{\varphi 0}$ is the physical dispersion due to the structure kick given in Eq. (B4) and $\eta_{\varphi A}$ is an artefact given by 
Eq. (B5). The artefact $\eta_{\varphi A}$ can be explained by considering that a change of the acceleration gradient of a tilted structure also changes the associated transverse kick which leads to an orbit change. While for the nominal gradient the beam orbit oscillation due to the transverse kick is corrected by the initial beam steering, this is not the case anymore for the changed transverse kick. This orbit change will be interpreted by the DFS algorithm as dispersion, which leads to a wrong dispersion correction and consequently to a strong performance degradation.

The effect is illustrated in Fig. 6, which shows the simulated DFS dispersion measurement in bin 25 . The only imperfection that has been applied is structure tilts with $\sigma_{\varphi}$ of $140 \mu \mathrm{rad}$. An initial beam steering has been performed. When determining $\eta_{\varphi}$, an energy change $\delta$ of 1 per mille has been created with a change of the accelerating gradients of the structures belonging to the decelerator before this bin (2986 structures of each $23 \mathrm{~cm}$ length). To be able to determine also the physical dispersion $\eta_{\varphi 0}$, the energy change $\delta$ of 1 per mille has been introduced artificially by changing the beam energy directly before the DFS bin. As already mentioned earlier, the physical dispersion $\eta_{\varphi 0}$ is small compared to the dispersion artefact $\eta_{\varphi A}$ in the correction bin. While $\eta_{\varphi 0}$ is a linear rising oscillation [see Eq. (15) for a general dipole kick], $\eta_{\varphi A}$ represents a steplike oscillation, namely the betatron oscillation.

For the full DFS dispersion measurement, not only the artefact $\eta_{\varphi A}$ of a single tilted structure has to be considered, but the combined artefact $\eta_{\varphi A}^{(\mathrm{DFS})}$ from all involved structures. The number of involved structures depends on how local the energy change $\delta$ is created, which can be characterised by the parameter

$$
r=\frac{N_{s}}{N_{g}}
$$

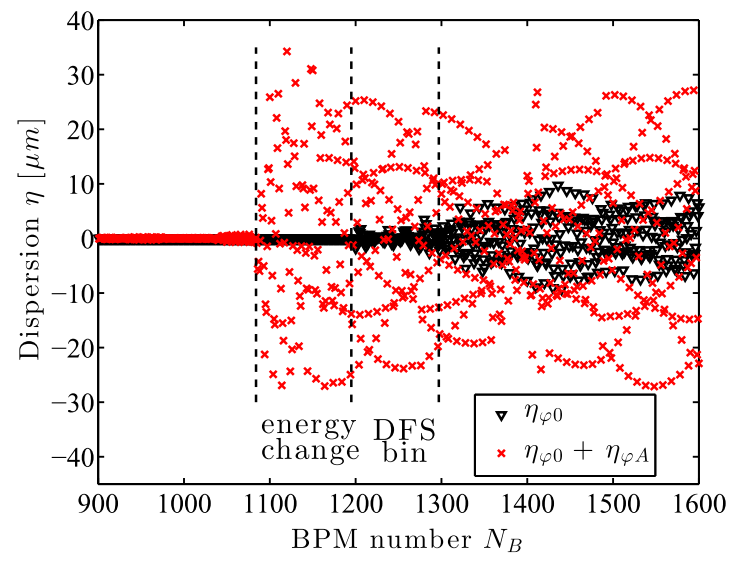

FIG. 6. Comparison of physical dispersion (black) and the dispersion measured (red) by the DFS algorithm, due to structure tilts in the main linac of CLIC. The data have been created with simulations for the DFS bin 25. where $N_{s}$ is the total number of structures before the correction bin and $N_{g}$ is the number of structures used to create the energy change $\delta$ by changing their gradients. Considering that the relative gradient change has to be $\hat{\delta}=r \delta$, the measured dispersion artefact $\eta_{\varphi A}^{(\mathrm{DFS})}$ is given by

$$
\eta_{\varphi A}^{(\mathrm{DFS})}=\sum_{i=1}^{N_{g}} \eta_{\varphi A}^{(i)} \propto \sqrt{N_{g}} \frac{\hat{\delta}}{\delta}=\sqrt{r} \sqrt{N_{s}} .
$$

Since the overall dispersion artefact scales as $\sqrt{N_{s}}$, the degrading effect will be stronger for bins further downstream. Also, the dispersion artefact is increased for schemes with more local energy change as it scales with $\sqrt{r}$.

\section{REALISTIC IMPLEMENTATION SCENARIOS FOR CLIC}

In this section, the correction performance and robustness properties of different variants of on-line DFS are simulated for the CLIC main linac. The simulation setup described in Sec. II E has been used as a realistic machine model that also implements the full beam-based alignment procedure described in Sec. II C.

In an accelerator that is powered by klystrons, the energy change $\delta$ necessary for dispersion measurement can be introduced very flexibly. The drive beam concept of CLIC has more restrictions in this respect. In the following, three options for CLIC are studied: global energy change, local energy change via the on/off mechanism of the power extraction and transfer structure (PETS) [26], and local energy change per drive beam decelerator. For the latter two options, additional adaptation to the basic dispersion estimation algorithm are necessary to cope with the increased sensitivity to structure tilts. These adaptations will be introduced below.

The performance of the three variants has been evaluated with respect to the most relevant imperfections, which are listed in Table I. The imperfections as well as the dispersion corrections are all applied in the horizontal as well as in the vertical direction. Since, the vertical emittance is much more sensitive than the horizontal one, only the correction

TABLE I. Standard deviation of the most relevant imperfections in horizontal and vertical plane for the main linac of CLIC considered for the on-line DFS studies.

\begin{tabular}{lcc}
\hline \hline Imperfection & Symbol & CLIC specification \\
\hline Quadrupole offset & $\sigma_{\mathrm{QP}}$ & $14 \mu \mathrm{m}$ \\
BPM offset & $\sigma_{\mathrm{O}}$ & $14 \mu \mathrm{m}$ \\
BPM resolution & $\sigma_{\mathrm{BPM}}$ & $50 \mathrm{~nm}$ \\
WFM resolution & $\sigma_{\mathrm{WFM}}$ & $3.5 \mu \mathrm{m}$ \\
Structure tilt & $\sigma_{\varphi}$ & $140 \mu \mathrm{rad}$ \\
\hline \hline
\end{tabular}


performance for the vertical direction will be only studied in this paper.

It should be mentioned that the quadrupole magnet itself is not a critical imperfection, since it is very well corrected after the beam steering already. For the effectiveness of the beam steering the BPM offset $\sigma_{\mathrm{O}}$ is much more relevant. In order to reduce the computation time for one simulation to about one hour, slightly simplified simulations have been performed, where the dispersion estimation process was implemented by determining the reference and off-energy orbits $x_{0}$ and $x_{\delta}$ and adding noise of the correct size $\tilde{\sigma}_{\mathrm{BPM}}$ according to Eq. (12). As described in Sec. II D, the number of averaged orbits for one measurement $N$ has been chosen to be 100 , which results in an effective BPM resolution $\tilde{\sigma}_{\mathrm{BPM}}$ of $7.07 \mathrm{~nm}$. The DFS parameter $\beta$ is set to 0 to not limit the correction performance, and $\omega$ will be chosen via the following simulation studies for each implementation scenario individually to minimize the remaining emittance growth.

\section{A. Global energy change}

The baseline beam-based alignment concept for CLIC foresees a DFS correction with a global beam energy change of 5\%. It can only be applied off-line, since beams with such energy deviations are lost in the collimation system of the beam delivery system. The beam energy change is introduced via two subsystems. The initial beam energy (entrance of main linac) is changed by varying the phase of the accelerating voltage of an upstream bunch compressor system [27]. The beam energy in the main linac is changed by varying the acceleration gradients of all accelerating structures. In CLIC, the gradient $G$ can be changed by adapting the drive beam charge $Q_{\mathrm{DB}}$, where $G$ is linearly dependent on $Q_{\mathrm{DB}}$ [28].

The performance of this global DFS scheme for an energy change $\delta$ of $5 \%$ is illustrated in Fig. 7. Each data point in the plot corresponds to an average over ten random seeds. The corresponding simulations have been carried out on the CERN parallel computing center, since each of the presented plots need about 360 hours calculation time on a standard computer.

Larger values of $\omega$ correspond to a stronger dispersion correction and BPM offset effects get corrected better. One the other hand, the parasitic BPM noise deteriorates the result with increasing $\omega$. However, for an effective BPM resolution of $7.07 \mathrm{~nm}$ hardly any emittance growth is observable, and an $\omega$ of about 50 is preferable. Also a correction without averaging can be considered (effective $\mathrm{BPM}$ resolution of $50 \mathrm{~nm}$ ), where an optimal value for $\omega$ is found to be about 2 . For structure tilts and the wakefield monitor resolution the result is independent of $\omega$, where the WFM resolution contributes stronger to the emittance growth. For $\omega_{\text {opt }}$, an average accumulated emittance growth of about $10 \%$ is expected, which shows that a global scheme with a $\delta$ of $5 \%$ is a good off-line DFS solution.

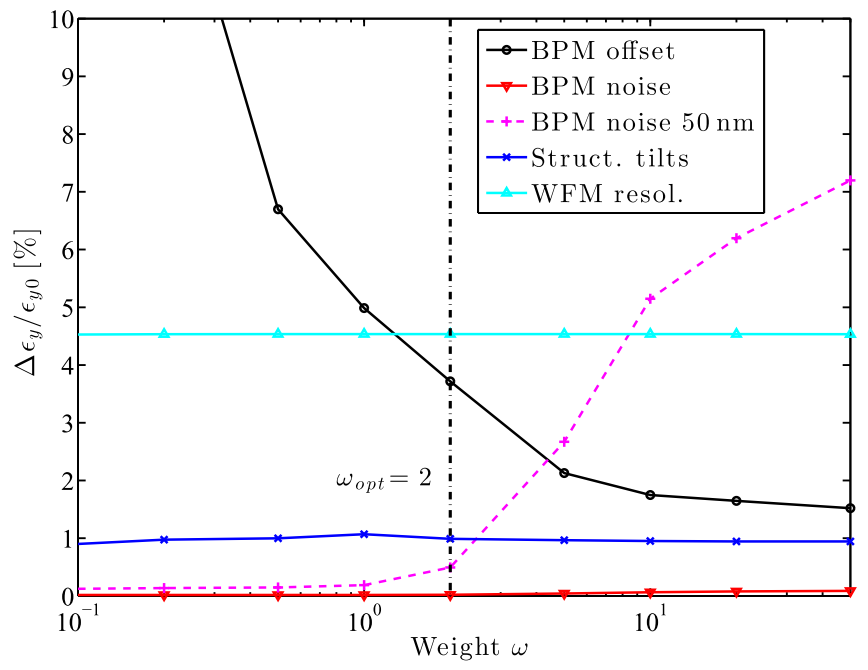

FIG. 7. DFS performance with respect to the vertical emittance growth $\Delta \epsilon_{y}$ relative to the nominal emittance $\epsilon_{0}$ of $10 \mathrm{~nm}$ for a scheme with a global energy change $\delta$ of $5 \%$ for different values of the DFS parameter $\omega$. The performance is evaluated for different imperfection, where an optimal weight $\omega_{\text {opt }}$ of about 2 has been found (vertical dashed line).

To be able to use the global scheme also for on-line DFS, the beam energy variation $\delta$ has to be reduced from 5 to $0.1 \%$. The result of such a decrease of $\delta$ is illustrated in Fig. 8. The simulations show that the sensitivity to wakefields is significantly increased, as has been predicted in Sec. III. For the CLIC specification $\sigma_{\mathrm{WFM}}$ of $3.5 \mu \mathrm{m}$, the performance for a global DFS scheme with a $\delta$ of $0.1 \%$ and even $1 \%$ is unsatisfactory. A global energy change scheme can hence not be used for the implementation of online DFS.

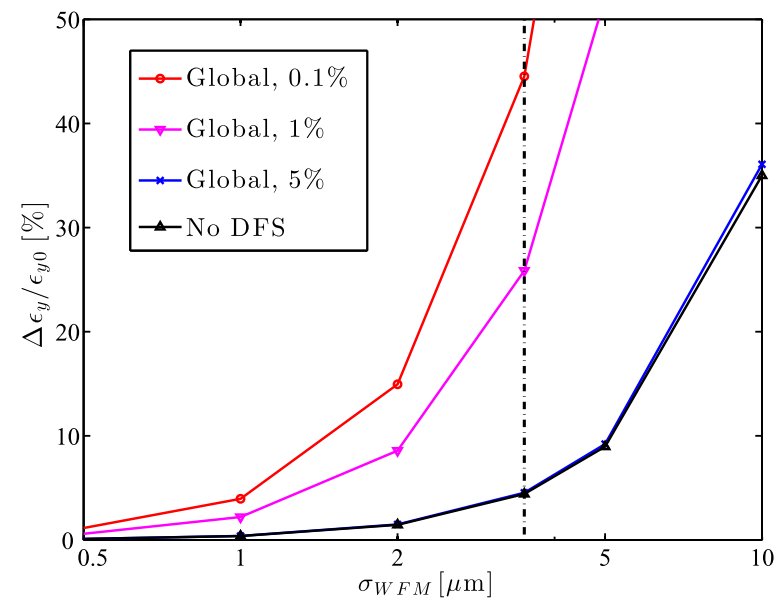

FIG. 8. Sensitivity of DFS to residual wakefields with respect to the vertical emittance growth $\Delta \epsilon_{y}$ relative to the nominal emittance $\epsilon_{0}$ of $10 \mathrm{~nm}$ for a scheme with a global energy change for different WFM resolutions. The CLIC specification for $\sigma_{\mathrm{WFM}}$ is $3.5 \mu \mathrm{m}$ and is indicated with the vertical dashed line. 


\section{B. PETS on/off option}

To reduce the wakefield sensitivity of a global DFS scheme, it was suggested in Sec. IIIE to create the necessary energy variation $\delta$ shortly before the correction bin. In this section, this idea is realized by completely turning off a few structures, just before the DFS bin with the help of the PETS on/off mechanism [26]. The PETS on/ off mechanism is a rf device that allows stopping the $\mathrm{rf}$ power flow from the PETS to the acceleration structures. In this scenario only a few structures are necessary to create the energy variation $\delta$ of $0.1 \%$.

The difficulty for this scheme is that due to the very local energy change the effect of structure tilt artefacts is severe. This can be predicted from Eq. (19) and has been verified in simulations. On the other hand, since only a few structures are turned off, mechanical alignment strategies can be envisioned. In the following two such options are studied.

The first option is to equip the few structures, which are used to create the energy difference, with two wakefield monitors instead of one. Since the specified accuracy of the wakefield monitors is $3.5 \mu \mathrm{m}$ and the distance between them is $0.25 \mathrm{~m}$, the structures could be aligned to a residual RMS tilt $\tilde{\sigma}_{\varphi}$ of $20 \mu \mathrm{rad}$, which is significantly better than the initial mechanical alignment $\sigma_{\varphi}$ of $140 \mu \mathrm{rad}$. However, simulation studies show that already for a $\tilde{\sigma}_{\varphi}$ of $10 \mu$ rad the vertical emittance growth $\Delta \epsilon_{y}$ is $98 \%$ after beam-based alignment (average over ten random seeds). To limit the emittance growth to $5 \%$ a $\tilde{\sigma}_{\varphi}$ of $2 \mu \mathrm{rad}$ is necessary, which shows this option cannot be used.

The second option that has been studied is to perform an initial mechanical tilt scan for the structures used to create the energy difference prior to the DFS correction. These scans are based on the fact that if a structure is not tilted then it does not create any dispersion artefacts and the dispersion in the first few BPMs of the bin is nearly zero. This is because the physical dispersion from other sources only rises slowly (linear regime). Two structures tilts $\varphi_{A}$ and $\varphi_{B}$ are scanned to minimize $\eta_{S}\left(\varphi_{A}, \varphi_{B}\right)$, which is the RMS of the dispersion in the first 10 BPMs. This corresponds to the minimization of the tilt artefact $\eta_{\varphi A}$. For the scan, the first and the last structure that are turned off are used to account for the phase advance difference between them. The tilts of the scanned structures compensate finally also the artefacts created by the other structures.

The scan is performed in 7 equal steps for each of the two structures. For each measurement, 100 orbits have to be averaged to achieve an effective BPM resolution of $7.07 \mathrm{~nm}$. This is necessary to reduce the associated emittance growth to below $0.5 \%$. With these parameters, the time for one scan is about one hour. Due to the limited resolution of only 49 data points and due to the remaining BPM noise a general second order function is fitted to the data with the least squares method. The minimum of this

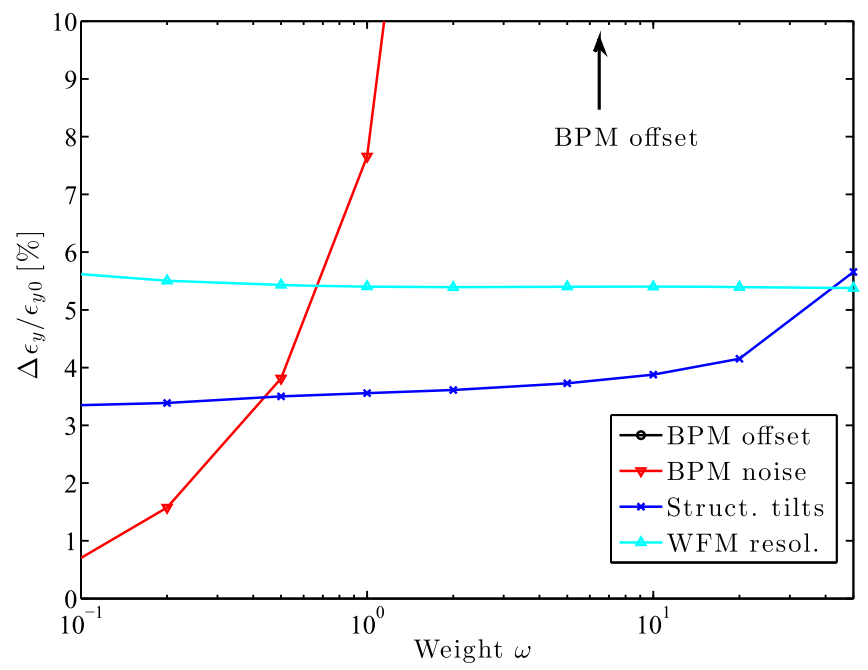

FIG. 9. DFS performance with respect to the vertical emittance growth $\Delta \epsilon_{y}$ relative to the nominal emittance $\epsilon_{0}$ of $10 \mathrm{~nm}$ as a function of the parameter $\omega$ for a scheme with a local energy change $\delta$ of $0.1 \%$ in each bin introduced by using the PETS on/off mechanism. The emittance growth for BPM offsets (black line) is unacceptably high and hence not visible in this plot.

fitted function corresponds to the structure tilt setting that minimizes the dispersion artefact.

The performance of the local DFS algorithm using the PETS on/off mechanism and including an initial tilt scan is shown in Fig. 9. As expected, the emittance growth due to the resolution of the wakefield monitors has been reduced to an acceptable level. Also, the sensitivity to structure tilts is low, due to the effectiveness of the tilt scans. However, especially the performance with respect to BPM offsets is dramatically worsened, such that the results are not even shown in the plot (500\% emittance growth). For this case, the tilt scans do not deliver accurate values for $\varphi_{A}$ and $\varphi_{B}$ anymore that minimize the tilt artefacts. The reason for this is that the physical dispersion due to BPM offsets is already large in the first $10 \mathrm{BPMs}$, and therefore the scans simultaneously minimize this physical dispersion and the tilt artefact. The corresponding tilt alignment is insufficient.

In summary, the DFS scheme using the PETS on/off mechanism and tilt scans is not an option for on-line DFS. Besides the insufficient performance with respect to BPM offsets, it has also several operational disadvantages as the long scanning time and the problematic mechanical actuation of the PETS on/off mechanism at the beam train repetition rate of $50 \mathrm{~Hz}$.

\section{Decelerator-based energy change}

The last section showed that an on-line DFS scheme that creates the energy change $\delta$ with the PETS on/off mechanism is hardly realizable. The reason for that is the structure tilt artefacts in the dispersion measurement, which are pronounced due to the very local energy change. A logical consequence of this finding is to use a less localised scheme 
in order to reduce the structure tilt artefacts, while still demagnifying wakefield effects compared to a global scheme. Such a scheme can be realized by changing the rf power produced by the decelerator before the correction bin, which at the same time changes the acceleration gradients in the 2976 supplied structures. Two options for such a scheme have been envisioned.

In the first option, the charge of the drive beam bunches that supply one decelerator is varied. Due to the recombination scheme of the drive beam [1] this corresponds to a specific bunch-to-bunch charge variation pattern within one drive beam train. The creation of this charge pattern with the required precision is difficult to realize. More promising is the second option that will be studied in the remainder of this section. Here, one decelerator is selectively supplied with one less drive beam bunch than the other decelerators. Such an option can be realized by a specific delayed switching scheme in the drive beam complex [29]. With this scheme the beam energy can only be lowered. The gradients of the corresponding accelerating structures are reduced by $4 \%$. In this scheme the relative energy variation $\delta_{i}$ is different for each DFS bin (beginning $\delta_{1}=4 \%$, end $\delta_{36}=0.17 \%$ ). The energy variation at the end of the main linac is however always $0.17 \%$. A completely transparent system could be realized in this fashion, by always running one of the decelerators at a lower energy and compensating for the energy loss by an $0.17 \%$ higher average drive beam charge.

Even though the structure tilt sensitivity of this scheme is significantly reduced compared to the PETS on/off option, it is still severe. Simulation studies show that structure tilts of $140 \mu \mathrm{rad}$ RMS (CLIC specification), cause a residual emittance growth $\Delta \epsilon_{y}$ of about $150 \%$. Hence, the decelerator-based scheme cannot be applied in its basic form. It is possible, however, to lower the structure tilt sensitivity, by considering the findings of Sec. IV. As visualized in Fig. 6, the measured dispersion $\eta$ is the sum of the physical dispersion $\eta_{R}$ and the artefact $\eta_{\varphi A}$ due to the structure tilts. In this case $\eta_{R}$ is not only the physical dispersion due to the structure kick, but contains also all other sources of dispersion as from quadrupole magnets and wakefields. The goal is now to find an estimate $\hat{\eta}_{\varphi A}$ for the structure tilt artefact, such that the measured dispersion can be corrected as

$$
\eta_{\text {corr }}=\eta-\hat{\eta}_{\varphi A}
$$

The measurement $\eta^{(i)}$ within the DFS bin $i$ contains a mixture of physical dispersion and artefacts. The corresponding data cannot be used to determine $\hat{\eta}_{\varphi A}$. However, in the area before the DFS bin only the dispersion artefact is present in the measurement $\eta^{(i-1)}$ (see Fig. 6), since the other sources of dispersion have been corrected already in the prior correction steps. To find an estimate $\hat{\eta}_{\varphi A}^{(i)}$ from the data $\eta^{(i-1)}$, one can exploit the fact that $\hat{\eta}_{\varphi A}$ has the form of a betatron oscillation given by Eq. (B5). Hence, virtual quadrupole magnet offsets $\Delta x_{i-1}$ can be found that create an oscillation that fit $\hat{\eta}_{\varphi A}^{(i-1)}$ in a least squares sense as

$$
\Delta x_{i-1}=R_{i-1 \rightarrow i-1}^{\dagger} \hat{\eta}_{\varphi A}^{(i-1)},
$$

where $R_{i-1 \rightarrow i-1}$ is the orbit response matrix due to quadrupole offset, and the symbol $\uparrow$ corresponds to the pseudoinverse. The betatron oscillation can then be propagated to the DFS bin, which gives the final estimate

$$
\hat{\eta}_{\varphi A}^{(i)}=R_{i-1 \rightarrow i} \Delta x_{i-1} .
$$

The performance of this modified decelerator-based DFS scheme is shown in Fig. 10. Each data point corresponds to an average of ten random seeds. Contrary to the PETS on/ off option, the correction performance for BPM offsets is at a satisfying level. Interestingly, for large values of $\omega$ the performance with respect to BPM offsets is worsened. This is due to the fact that the found DFS orbit is relatively far from the initial orbit after beam steering to which the structures have been aligned via the rf alignment. The corresponding additional wakefield effects are only corrected at the end of every iteration, but not after each bin correction to limit the overall correction time. The corresponding wakefield effects deteriorate the DFS correction before they are corrected. This effect is only relevant for BPM offsets, since the other imperfections create much smaller orbit variation via the DFS correction. The optimal weight $\omega_{\text {opt }}$ is found to be about 1 , which is a trade-off between the sensitivity to BPM offsets and BPM noise.

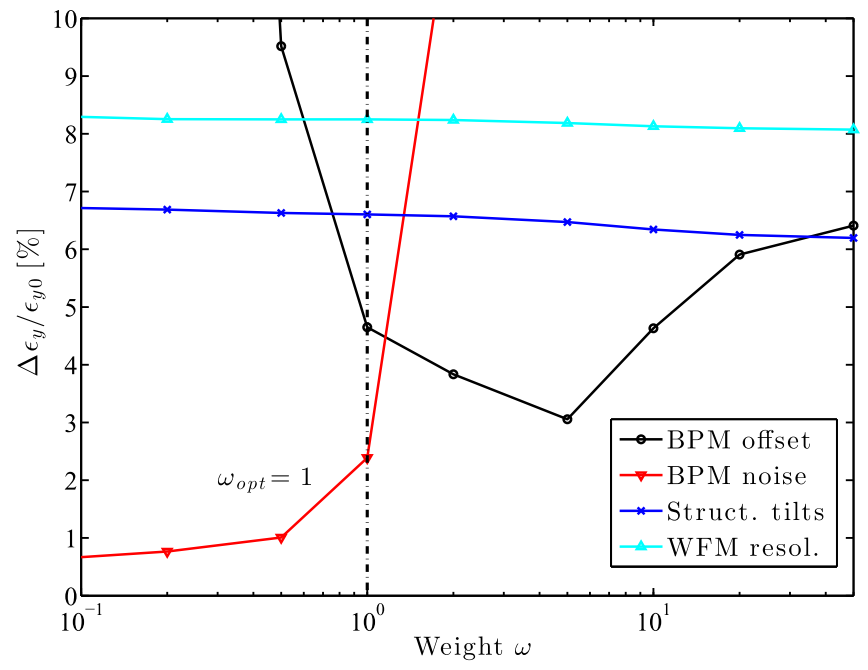

FIG. 10. DFS performance with respect to the vertical emittance growth $\Delta \epsilon_{y}$ relative to the nominal emittance $\epsilon_{0}$ of $10 \mathrm{~nm}$ as a function of the DFS parameter $\omega$ for a scheme with a local energy change $\delta$ created by changing the accelerating gradients of structures belonging to one decelerator by $4 \%$. The performance is evaluated for different imperfection, where an optimal weight $\omega_{\text {opt }}$ of about 1 has been found (vertical dashed line). 
The sensitivity to wakefields is worsened compared to the PETS on/off option, due to the less localized energy change. Also the sensitivity to structure tilts is increase compared to the PETS on/off option, due to the less efficient structure tilt artefact suppression. For $\omega_{\mathrm{opt}}$, an average accumulated emittance growth of about $22 \%$ is expected, which shows that the presented scheme can be used for on-line DFS correction. The parameter space for an acceptable value of $\omega$ is rather narrow. An increase of the averaging time could widen this parameter space, due to a reduced effective BPM resolution $\tilde{\sigma}_{\mathrm{BPM}}$. Also, preliminary studies show that by varying the weight $\omega$ for different bins, the result can be further improved.

\section{ADDITIONAL PERFORMANCE AND ROBUSTNESS STUDIES}

The last section has shown that only one investigated variant of DFS can be used for an on-line application. This scheme creates a local energy change by changing the acceleration gradients of the structures belonging to one decelerator by $4 \%$. In this section, the performance and the robustness properties of this scheme are studied in more detail. For these additional results, the earlier found optimal weight $\omega_{\text {opt }}$ of 1.0 has been used.

The influence of the mechanical roll of BPMs and quadrupole magnet has been investigated via simulations by introducing these imperfections separately to the beamline. Gaussian distributed rolls with a standard deviation of $100 \mu \mathrm{rad}$ have been applied, which corresponds to the CLIC alignment specification. Since coupling effects due to element rolls are stronger when the beam orbit is larger, also BPM offsets in both planes according to Table I have been included in the beamline setup in order to obtain relevant results. If only BPM offsets are introduced, the vertical emittance growth after the DFS correction is $4.8 \%$ (average over 20 random seeds). If additionally BPM rolls are included, the change in emittance growth is $<0.1 \%$. Also, quadrupole magnet rolls cause an insignificant emittance variation of only about $0.2 \%$ and can hence be neglected with respect to the on-line DFS correction.

In Fig. 11, the local on-line DFS algorithm scheme is utilized for correcting a set of imperfections that corresponds to the initial mechanical alignment after the linac installation. All imperfections in Table I as well as $100 \mu \mathrm{rad}$ BPM and quadrupole magnets rolls are included in the simulations. The probability $\mathrm{p}\left(\Delta \epsilon_{y}>\Delta \epsilon_{y, 0}\right)$ is plotted on the vertical axis, which corresponds to the percentage of machines that do not reach the correction performance given on the horizontal axis. The shown results are created by evaluating 200 random seeds. The correction performance after each of the three iterations of the local scheme ( $4 \%$ gradient change per decelerator) is compared with the three iterations of the global scheme with an energy change of $5 \%$.

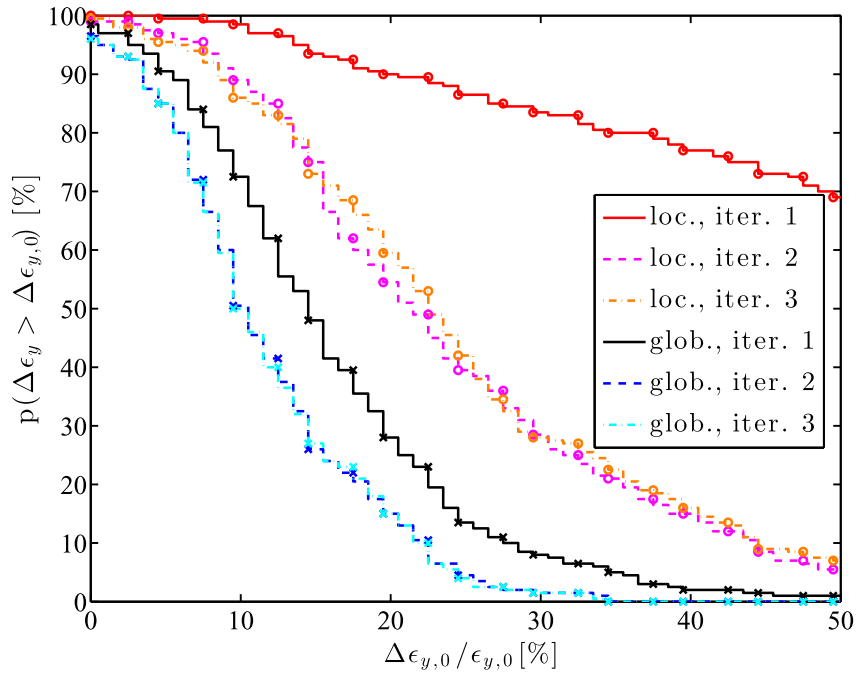

FIG. 11. Probability $\mathrm{p}$ of not reaching a certain correction level after the DFS correction, where the values are given relative to the nominal emittance $\epsilon_{0}$ of $10 \mathrm{~nm}$. The static imperfections in Table I as well as BPM and quadrupole magnet rolls are included. Two correction schemes are tested: the local scheme with a change the acceleration gradients per decelerator of $4 \%$ (loc.), and the global scheme with an acceleration gradient change of 5\% (glob.). The results after each of the three iterations of the corrections are shown.

After the first iteration of the local scheme the emittance is not corrected sufficiently, since the CLIC specifications require that $90 \%$ of the machines are corrected better than to an emittance growth of $50 \%$. This goal is clearly met after the second iteration, while the third iteration does not improve the performance any further. On average, the emittance growth is $23 \%$. The global scheme performs even better than the local one. Already after the first iteration the CLIC specification is clearly met. While the second iteration still improves the result, also here the third iteration creates only insignificant changes. The average emittance increase is as small as $11 \%$. The global scheme clearly performs better than the local one, but it cannot be used in an on-line application. One the other hand, the local scheme also clearly reaches the CLIC specification.

Also the effect of dynamic imperfections has been studied. For the imperfections tested so far, the dispersion could be efficiently simulated by determining the reference and off-energy orbits $x_{0}$ and $x_{\delta}$ and adding scaled noise of the correct size. For dynamic studies, this approach is not applicable. Instead, the full estimation process has been implemented. As a test scenario for the dynamic imperfections, an on-line DFS correction after 12 hours without any correction has been considered. The according results are summarized in Table II, where each emittance growth value corresponds to an average over 20 random seeds. The values of the applied dynamic imperfections are in accordance with the CLIC baseline specifications.

If uncorrected, the component misalignment due to ATL ground motion causes an emittance growth of $79 \%$, which 
TABLE II. Relative vertical emittance growth $\Delta \epsilon_{y} / \epsilon_{y, 0}$, where $\epsilon_{0}$ is the nominal emittance of $10 \mathrm{~nm}$, after on-line DFS correction including different dynamic imperfections in the dispersion estimation process: initial ATL misalignments, BPM noise, orbit feedback system (O-FB), short term ground motion (GM), random accelerating gradient errors coherent for one decelerator $\Delta G_{d}$ or for the whole main linac $\Delta G_{l}$. Each relative emittance growth value is an average over 20 random seeds.

\begin{tabular}{lcc}
\hline \hline Imperfections & Value/Type & $\Delta \epsilon_{y} / \epsilon_{y, 0}$ \\
\hline ATL $(T=12 \mathrm{~h})$ & $A=10^{-5} \mu \mathrm{m}^{2} / \mathrm{m} / \mathrm{s}[19]$ & $0.2 \%$ \\
ATL, BPM & $\sigma_{\mathrm{BPM}}=50 \mathrm{~nm}$ & $2.8 \%$ \\
ATL, BPM, O-FB & O-FB according to [30] & $3.8 \%$ \\
ATL, BPM, O-FB, GM & model B10 [31] & $5.5 \%$ \\
ATL, BPM, O-FB, $\Delta G_{d}$ & $\Delta G_{d} / G_{d, 0}=5 \times 10^{-3}$ & $3.4 \%$ \\
ATL, BPM, O-FB, $\Delta G_{l}$ & $\Delta G_{l} / G_{d, 0}=10^{-3}$ & $3.5 \%$ \\
\hline \hline
\end{tabular}

is in good agreement with the empirical model in Sec. I that predicts an emittance growth of $90 \%$. The application of on-line DFS mitigates this misalignment effect nearly completely to a residual value $\Delta \epsilon_{y} / \epsilon_{y, 0}$ of $0.2 \%$. If also BPM noise is included in the simulations, the residual emittance growth increased to $2.8 \%$, which is very good agreement with the value predicted by the simplified simulations in Sec. VC. The additional application of the orbit feedback system contributes to another $1 \%$ of emittance increase, and short time ground motion according to the model B10 [31] increases the residual emittance growth to $5.5 \%$. Model B10 is the baseline ground motion model for CLIC. It is a modified version of the well-known model B [32], where the high-frequency components have been amplified by a factor of ten.

Also two types of acceleration gradient errors during the estimation process have been tested: a gradient error $\Delta G_{l}$ that is coherent along the machine put different from beam train to beam train, and a gradient error $\Delta G_{d}$ that is only coherent along one decelerator, but different from decelerator to decelerator and from beam train to beam train. Both effects have only a small effect and can be neglected. The emittance growth with these energy errors is even slightly smaller than without them, which can be explained by the limited statistic of 20 random seeds.

\section{CONCLUSIONS}

In this paper, the problem of emittance growth in the CLIC main linac due to the dynamic component misalignments via ground motion on the minutes to hours scale is addressed. To mitigate this effect, an on-line dispersion estimation and correction scheme has been proposed that is based on the well-known DFS algorithm. By lowering the beam energy change $\delta$ for the necessary dispersion measurement to the per mille level, the dispersion measurement is not disturbing the usual accelerator operation. The higher sensitivity with respect to BPM noise, beam energy jitter, and short-term ground motion can be overcome with an averaging strategy, where several noisy measurements are combined to one of sufficient quality. Also, the sensitivity to residual transverse wakefields and structure tilts is increased. Analytical models have been developed that are capable of explaining these effects and mitigation strategies have been suggested.

As a result, a practically applicable on-line dispersion correction scheme for the main linac of CLIC could be proposed. In this scheme, the necessary beam energy change $\delta$ is introduced by a $4 \%$ acceleration gradient variation in only $4 \%$ of the main linac structures, namely in the ones belonging to the decelerator directly before the correction bin (2986 structures each $23 \mathrm{~cm}$ long). Simulation studies show that the scheme is robust with respect to all critical imperfections. The average emittance growth due to static misalignments after beam-based alignment is about $22 \%$. The full correction takes 4 minutes and 16 second. This is well within the emittance budget of $50 \%$. The simulations also indicate that a variable DFS parameter choice for different bins along the accelerator can further improve this result, which is subject to future work. Also dynamic component misalignment can be very effectively mitigated. The associated emittance growth after correction is only $5.5 \%$, even if pulse-to-pulse energy jitter, short-term ground motion and the orbit feedback system are included in the simulations.

In summary, the presented on-line dispersion measurement and correction scheme provides an effective countermeasure for the up to now unsolved problem of dynamic component misalignments on the minutes scale. Since the solution of this problem is critical for the operation of CLIC, this scheme is likely become an essential operational tool. Additionally, it is also of interest for the dispersion correction in the beam delivery systems, where due to the limited energy acceptance of the collimation systems only small energy changes can be introduced. Apart from the application to CLIC, the sensitivity analysis presented in this paper is also of general interest for the application of DFS with small energy changes. In the future, it would be certainly desirable to measure the effects found via the simulations in this work in a real machine.

\section{ACKNOWLEDGMENTS}

The authors would like to thank Steffen Döbert and Carl Lindstrøm for the valuable discussions with them. This work is supported by the Research Council of Norway.

\section{APPENDIX A: DISPERSION MODEL FOR BEAMLINES}

The dispersion $\eta(s)$ at position $s$ of long beamline is defined as

$$
\eta(s)=\frac{x_{\delta}(s)-x_{0}(s)}{\delta}
$$


were $x_{\delta}(s)$ and $x_{0}(s)$ are the trajectories of two particles with energies $E_{0}(1+\delta)$ and $E_{0}$, where $\delta \ll 1$. When only a single dipole kick is considered, the particle trajectory $x_{0}(s)$ is described by the inhomogeneous differential equation

$$
x_{0}^{\prime \prime}(s)+k_{0}(s) x_{0}(s)=\Delta x_{0}^{\prime} \delta_{k}\left(s-s_{k}\right),
$$

where $\delta_{k}\left(s-s_{k}\right)$ is a Dirac distribution peaking at position $s_{k}$, and $\Delta x_{0}^{\prime}$ is the amplitude of a lumped kick. The wellknown solution of Eq. (A2) is the sum of the homogeneous and a particular solution. For simplicity, the initial conditions can be set to $x_{0}\left(s_{0}\right)=0$ and $x_{0}^{\prime}\left(s_{0}\right)=0$, which results in the solution

$$
\begin{aligned}
& x_{0}\left(s<s_{k}\right)=0 \quad \text { and } \\
& x_{0}\left(s \geq s_{k}\right)=\sqrt{\beta_{0}\left(s_{k}\right) \beta_{0}(s)} \sin \left[\hat{\phi}_{0}(s)\right] \Delta x_{0}^{\prime}
\end{aligned}
$$

with

$$
\hat{\phi}_{0}(s)=\phi_{0}(s)-\phi_{0}\left(s_{k}\right) .
$$

The expressions $\beta_{0}(s)$ and $\phi_{0}(s)$ are the $\beta$-function and the phase advance of a particle with energy $E_{0}$, respectively.

To calculate the trajectory $x_{\delta}(s)$ of the off-momentum particle, $k_{0}(s)$ and $\Delta x_{0}^{\prime}$ have to be scaled to the energy $E_{0}(1+\delta)$, which results in the equation

$$
x_{\delta}^{\prime \prime}(s)+\frac{k_{0}(s)}{1+\delta} x_{\delta}(s)=\frac{\Delta x_{0}^{\prime}}{1+\delta} \delta_{k}\left(s-s_{k}\right),
$$

with the analogous solution

$$
\begin{aligned}
& x_{\delta}\left(s<s_{k}\right)=0 \quad \text { and } \\
& x_{\delta}\left(s \geq s_{k}\right)=\sqrt{\beta_{\delta}\left(s_{k}\right) \beta_{\delta}(s)} \sin \left[\hat{\phi}_{\delta}(s)\right] \frac{\Delta x_{0}^{\prime}}{1+\delta}
\end{aligned}
$$

with

$$
\hat{\phi}_{\delta}(s)=\phi_{\delta}(s)-\phi_{\delta}\left(s_{k}\right) .
$$

Also here, $\beta_{\delta}(s)$ and $\phi_{\delta}(s)$ are the $\beta$-function and phase advance of a particle with energy $E_{0}(1+\delta)$, respectively. Substituting Eqs. (A3)-(A6), into Eq. (A1), the dispersion $\eta(s)$ is found to be

$$
\begin{aligned}
\eta\left(s>s_{k}\right)= & \sqrt{\beta_{\delta}\left(s_{k}\right) \beta_{\delta}(s)} \sin \left[\hat{\phi}_{\delta}(s)\right] \frac{\Delta x_{0}^{\prime}}{(1+\delta) \delta} \\
& -\sqrt{\beta_{0}\left(s_{k}\right) \beta_{0}(s)} \sin \left[\hat{\phi}_{0}(s)\right] \frac{\Delta x_{0}^{\prime}}{\delta} .
\end{aligned}
$$

To further simplify this expression, the energy scaling of the $\beta$-function in a FODO lattice for small $\delta$ can be used [33], which is given by

$$
\beta_{\delta}(s) \approx \beta_{0}(s)(1+\delta) .
$$

Exploiting this fact, Eq. (A8) can be written as

$$
\begin{gathered}
\eta\left(s>s_{k}\right)=\sqrt{\beta_{0}\left(s_{k}\right) \beta_{0}(s)} T(s) \frac{\Delta x_{0}^{\prime}}{\delta} \quad \text { with } \\
T(s)=\sin \left[\hat{\phi}_{\delta}(s)\right]-\sin \left[\hat{\phi}_{0}(s)\right] .
\end{gathered}
$$

This last step corresponds to the physical fact that the amplitudes of the oscillations $x_{\delta}$ and $x_{0}$ are the same. The dispersion $\eta$ is hence only built up due to the phase difference of the two oscillations given in Eq. (A11). The dispersion expression can be further simplified, since the phase advance of the particle with higher energy $\hat{\phi}_{\delta}(s)$ only slightly deviates from $\hat{\phi}_{0}(s)$. This suggests to write $\hat{\phi}_{\delta}(s)$ as

$$
\hat{\phi}_{\delta}(s)=\Delta \hat{\phi}_{\delta}(s)+\hat{\phi}_{0}(s)
$$

where $\Delta \hat{\phi}_{\delta}(s)$ is the phase deviation due to the energy difference. If $\Delta \hat{\phi}_{\delta}(s) \ll 1$, Eq. (A11) can be approximated as

$$
T(s) \approx-\cos \left[\hat{\phi}_{0}(s)\right] \Delta \hat{\phi}_{\delta}(s),
$$

where trigonometric identities and a truncated Taylor series expansion have been used. The phase difference $\Delta \hat{\phi}_{\delta}(s)$ is given by

$$
\Delta \hat{\phi}_{\delta}(s)=\hat{\xi}(s) \delta
$$

where $\hat{\xi}(s)$ is the chromaticity between $s$ and $s_{k}$. This chromaticity can be approximated with the expression for the chromaticity of a single FODO cell $\xi_{F}$ as

$$
\hat{\xi}(s) \approx \xi_{F} \frac{\hat{N}_{q}(s)}{2},
$$

where $\hat{N}_{q}(s)$ is the number of quadrupole magnets between $s$ and $s_{k}$, and $\xi_{F}$ is given by [[34] p. 76]

$$
\xi_{F}=-2 \tan \left(\frac{\hat{\phi}_{0, F}}{2}\right)
$$

where $\hat{\phi}_{0, F}$ is the phase advance of one FODO cell. Finally, the dispersion due to a single dipole kick $\Delta x_{0}^{\prime}$ for $\Delta \hat{\phi}_{\delta}(s) \ll 1$ can be expressed as

$$
\eta_{L}(s)=\sqrt{\beta_{0}\left(s_{k}\right) \beta_{0}(s)} \cos \left[\hat{\phi}_{0}(s)\right] \frac{\xi_{F} \hat{N}_{q}(s)}{2} \Delta x_{0}^{\prime} .
$$

\section{APPENDIX B: MODEL OF DISPERSION ARTEFACT DUE TO STRUCTURE TILTS}

The signal measured due to one tilted acceleration structure can be modeled by evaluating the individual orbits $x_{0}$ and $x_{\delta}$ in Eq. (A1) for the corresponding dipole kick in Eq. (17). For this case, the orbit for the nominal beam energy is 


$$
x_{0}\left(s \geq s_{k}\right)=\sqrt{\beta_{0}\left(s_{k}\right) \beta_{0}(s)} \sin \left[\hat{\phi}_{0}(s)\right] \Delta x_{\varphi}^{\prime} .
$$

For the off-energy orbit $x_{\delta}$, it has to be considered that not only the beam energy is changed to $E_{0}(1+\delta)$, but also the transverse kick $\Delta x_{\varphi}^{\prime}$ from the tilted structure is changed to $\Delta x_{\varphi}^{\prime}(1+\tilde{\delta})$, since the acceleration gradient has been changed to introduce the beam energy change. Hence, $x_{\delta}$ is given by

$x_{\delta}\left(s \geq s_{k}\right)=\sqrt{\beta_{\delta}\left(s_{k}\right) \beta_{\delta}(s)} \sin \left[\hat{\phi}_{\delta}(s)\right] \frac{\Delta x_{\varphi}^{\prime}(1+\tilde{\delta})}{1+\delta}$.

In a scheme with a global beam energy change $\delta=\tilde{\delta}$, while for a local energy change $\delta<\tilde{\delta}$. The dispersion $\eta_{\varphi}$ can now be written as

$$
\eta_{\varphi}=\eta_{\varphi 0}+\eta_{\varphi A}
$$

where $\eta_{\varphi 0}$ is the physical dispersion due to the structure kick, which is given by

$\eta_{\varphi 0}=\sqrt{\beta_{0}\left(s_{k}\right) \beta_{0}(s)}\left\{\sin \left[\hat{\phi}_{\delta}(s)\right]-\sin \left[\hat{\phi}_{0}(s)\right]\right\} \frac{\Delta x_{\varphi}^{\prime}}{E_{0} \delta}$,

and $\eta_{\varphi A}$ is an artefact of the form

$$
\eta_{\varphi A}=\sqrt{\beta_{0}\left(s_{k}\right) \beta_{0}(s)} \sin \left[\hat{\phi}_{\delta}(s)\right] \frac{\Delta x_{\varphi}^{\prime} \tilde{\delta}}{E_{0} \delta} .
$$

[1] CLIC Conceptual Design Report, Volume: "A multi-TeV Linear Collider based on CLIC Technology," http://projectclic-cdr.web.cern.ch/project-clic-cdr/CDR_Volume1.pdf, 2012.

[2] T. O. Raubenheimer, Estimates of emittance dilution and stability in high-energy linear accelerators, Phys. Rev. ST Accel. Beams 3, 121002 (2000).

[3] K. Kubo, Estimation of orbit change and emittance growth due to random misalignment in long linacs, Phys. Rev. ST Accel. Beams 14, 014401 (2011).

[4] A. Sery and A. Mosnier, Spectral analysis of correction techniques for linear colliders, Phys. Rev. E 56, 3558 (1997).

[5] T. O. Raubenheimer and P. Tenenbaum, SLAC Report No. SLAC-TN-03-071, 2004.

[6] The Stanford Linear Collider, SLAC Report No. SLACPUB-5607, 1991.

[7] T. O. Raubenheimer and R. D. Ruth, A dispersion-free trajectory correction technique for linear colliders, Nucl. Instrum. Methods Phys. Res., Sect. A 302, 191 (1991).

[8] Ch. Adolphson and T. O. Raubenheimer, Method to evaluate steering and alignment algorithms for controlling emittance growth, in Proceedings of the 15th Particle Accelerator Conference, PAC-1993, Washington, DC, 1993 (IEEE, New York, 1993), p. 417.
[9] International Linear Collider Reference Design Report, Volume 3: Accelerator, https://www.linearcollider.org/ILC/ TDR, 2007.

[10] J. C. Smith, L. Gibbons, R. Patterson, D. Rubin, D. Sagan, and P. Tenenbaum, Comparison of beam-based alignment algorithms for the ILC, in Proceedings of the 21st Particle Accelerator Conference, Knoxville, TN, 2005 (IEEE, Piscataway, NJ, 2005), p. 1847.

[11] D. Schulte, Different options for dispersion free steering in the CLIC main linac, in Proceedings of the 21st Particle Accelerator Conference, Knoxville, TN, 2005 (IEEE, Piscataway, NJ, 2005) p. 1251.

[12] A. Latina and P. Raimondi, A novel alignment procedure for the final focus of future linear colliders, in Proceedings of the 25th International Linear Accelerator Conference, LINAC-2010, Tsukuba, Japan (KEK, Tsukuba, Japan, 2010), MOP026.

[13] T. O. Raubenheimer, A new technique of correcting emittance dilutions in linear colliders, Nucl. Instrum. Methods Phys. Res., Sect. A 306, 61 (1991).

[14] R. Assmann, T. Chen, F. J. Decker, M. Minty, T. Raubenheimer, and R. Siemann, Quadrupole alignment and trajectory correction for future linear colliders: SLC tests of a dispersion-free steering algorithm, in Proceedings of the 4th International Workshop on Accelerator Alignment, Tsukuba, 1995 (KEK, Tsukuba, 1995), p. 463.

[15] R. Assmann, P. Raimondi, G. Roy, and J. Wenninger, Emittance optimization with dispersion free steering at LEP, Phys. Rev. ST Accel. Beams 3, 121001 (2000).

[16] FACET Conceptual Design Report, SLAC Report No. SLAC-R-930, 2009.

[17] A. Latina, J. Pfingstner, D. Schulte, E. Adli, F. J. Decker, and N. Lipkowitz, Experimental demonstration of a global dispersion-free steering correction at the new linac test facility at SLAC, Phys. Rev. ST Accel. Beams 17, 042803 (2014).

[18] D. Schulte, Emittance Preservation in the Main LINAC of CLIC, in Proceedings of the 6th European Particle Accelerator Conference, Stockholm, 1998 (IOP, London, 1998), p. 478.

[19] V. Shiltsev, Observation of Random Walk of the Ground in Space and Time, Phys. Rev. Lett. 104, 238501 (2010).

[20] K. J. Åström and B. Wittenmark, Adaptive Control (Dover Publications, New York, 2008).

[21] Y. Sun and Ch. Adolphsen, Report No. SLAC-PUB-14323, 2011.

[22] N. Leros and D. Schulte, Static beam-based alignment of the RF structures in the CLIC main linac, in Proceedings of the 8th European Particle Accelerator Conference, Paris, 2002 (EPS-IGA and CERN, Geneva, 2002), p. 461.

[23] The tracking code PLACET, https://clicsw.web.cern.ch/ clicsw/.

[24] J. Snuverink, N. Fuster-Martinez, and J. Pfingstner, A linear accelerator simulation framework, in Proceedings of the 6th International Particle Accelerator Conference, Richmond, 2015 (JACoW, Geneva, 2015), MOPJE029.

[25] Octave https://www.gnu.org/software/octave.

[26] I. Syratchev and A. Cappellatti, A new high-power RF device to vary the output power of CLIC power extraction and transfer structures (PETS), in Proceedings of the 
International Particle Accelerator Conference, Kyoto, Japan (ICR, Kyoto, 2010), p. WEPE026.

[27] A. Latina, D. Schulte, and P. Eliasson, Bunch compressor for beam-based alignment, in Proceedings of the 22nd Particle Accelerator Conference, PAC-2007, Albuquerque, NM (IEEE, New York, 2007), p. THPMN061.

[28] I. Syratchev, PETS and drive beam development for CLIC, in X-Band RF Structure and Beam Dynamics Workshop 2008 (JACoW, Geneva, 2008), http://indico.cern.ch/event/ 39372/contributions/1829823/attachments/787975/ 1080123/X_band_2008_PETS.pdf.

[29] D. Schulte and I. Syratchev, Beam loading compensation in the main linac of CLIC, in Proceedings of the 20th International Linac Conference, LINAC-2000, Monterey, CA, 2000 (SLAC, Menlo Park, CA, 2000), p. MOA04.
[30] J. Pfingstner, Ph.D. thesis, Vienna University of Technology, 2013.

[31] J. Pfingstner, J. Snuverink, and D. Schulte, Ground motion optimized orbit feedback design for the future linear collider, Nucl. Instrum. Methods Phys. Res., Sect. A 703, 168 (2013).

[32] A. Sery and O. Napoly, Influence of ground motion on the time evolution of beams in linear colliders, Phys. Rev. E 53, 5323 (1996).

[33] D. Schulte, Lattice design considerations, in Proceedings of the 4th International Accelerator School for Linear Collider, Beijing, 2009 (JACoW, Geneva, 2009).

[34] A. Chao and M. Tigner, Handbook of Accelerator Physics and Engineering (World Scientific Publishing, Singapore, 1999), ISBN: 981-02-3500-3. 